\title{
Patient expectations of podiatric surgery in the United Kingdom
}

\author{
Antony N Wilkinson ${ }^{1}$ and Anthony J Maher ${ }^{2^{*}}$
}

\begin{abstract}
Background: Patient expectations can be difficult to conceptualise and are liable to change with time, health and environmental factors. Patient expectation is known to influence satisfaction, however little is known about the expectations of patients attending for podiatric surgery. This paper will explore the expectations of a large cohort of patients undergoing elective foot surgery.

Methods: The UK based podiatric audit of surgery and clinical outcome measurement (PASCOM) audit system was applied to a consecutive cohort of patients undergoing elective podiatric surgery in Doncaster, South Yorkshire between 2004 and 2010. Data was collected relating to the surgical episode and patient expectations. A patient questionnaire was administered at 6 months post intervention.
\end{abstract}

Results: A total of 2910 unique surgical admissions were completed and satisfaction questionnaires were returned by 1869 patients. A total of 1430 patients answered question 1 which relates to patient expectations. Pain relief was the most frequent expectation with 1191 counts (52.3\%), while footwear and mobility accounted for 16.6\% and $16.4 \%$ respectively. Cosmesis counts occurred less commonly; $12.2 \% .709$ patients (49.6\%) stated only a single expectation, 599 patients (41.9\%) stated two expectations, 114 patients (8\%) stated three expectations and 7 patients $(0.5 \%)$ stated 4 expectations. Pain relief was the dominant expectation accounting for 515 counts (72.6\%) of patients who provided only one response.

Conclusions: This paper demonstrates the expectations of a large cohort of podiatric surgery patients. For the most part patients expect pain relief, improved mobility and improved shoe fitting, while a small number of patients also expect a cosmetic improvement. Further research is required to determine the relationship between patient expectation and health related quality of life, and to determine whether podiatric surgery is successful in addressing the expectations of patients.

\section{Background}

Within healthcare there has, in recent years, been a shift in research and audit towards the assessment of patient expectations, satisfaction and outcomes, as opposed to pure clinical measurements [1]. In the United Kingdom, the sea change can be traced back to 1983 when the National Health Service (NHS) Management Enquiry condemned the NHS for its failure to use market research techniques to evaluate service provision [2].

Patient expectations are related to satisfaction and satisfaction itself is best considered a consequence of successfully meeting a patient's expectations. Patient

\footnotetext{
* Correspondence: anthonymaher@live.co.uk

${ }^{2}$ Department of Podiatric Surgery, Park House Health and Social Care Centre,

61 Burton Road, Carlton, Nottingham, NG4 3DQ, UK

Full list of author information is available at the end of the article
}

expectations can be difficult to conceptualise and are liable to change with time, health and environmental factors. Accepting the variability of patient expectation, satisfaction cannot be assessed in isolation without reference to the pre treatment expectations $[3,4]$.

Patient expectations may directly influence satisfaction with the service provided. In evaluating satisfaction with a primary care out of hours services, Mckinley et al state that patients who receive the care they hoped for were more satisfied [5]. It is recognised that patient expectations are an important independent predictor of success or failure of total joint arthroplasty [6] and high but realistic expectations are associated with improved orthopaedic surgery outcomes [7-12]. The reverse is also true, in that unrealistically high expectations may adversely influence the outcome of surgery $[7,13,14]$.

\section{() Biomed Central}


Within podiatric surgery authors have reported patient satisfaction as an outcome of intervention ([15-20]. Strangely though, there is little comment about patient expectations prior to elective foot surgery. A Medline search reveals only two previous papers expressly considering patient expectation in the context of hallux valgus surgery $[21,22]$.

David Tollafield and Gavin Rudge developed a patient satisfaction questionnaire (PSQ) in the 1990's expressly for use in podiatric surgery [23,24]. The PSQ was developed as a component of the Podiatric Audit of Surgery and Clinical Outcome Measures (PASCOM) instrument which is an ongoing audit project in the United Kingdom tasked with data collection in podiatry. The system initially collected surgical activity data, including satisfaction scores through a Microsoft Access database which was analysed both locally and nationally $[18,23]$.

The Patient satisfaction questionnaire (PSQ) asks a series of between 10 and 14 questions relating to a patient's experience of an episode of care (Additional File 1). There are a number of possible weighted answers for questions 2 to 10 and the scores for each are totalled with a maximum possible score of 100 and a minimum possible score of 0 . The total score (PATSAT score) is said to be a reflection of the patient's satisfaction with their surgery experience [23-25].

Question one of the PSQ sits apart from its neighbours because it requires a free text response from the patient. Additionally there is no scoring or weight attached to it and as such question one is excluded from the summary scoring playing no part in the final PATSAT score. There has been surprisingly little mention of question one in podiatric surgery reports. This paper will utilise question one of the PSQ to explore the aims and expectations of a large cohort of patients undergoing Podiatric Surgery.

\section{Methods}

The PASCOM audit system was applied to a consecutive cohort of patients undergoing elective podiatric surgery in Doncaster, South Yorkshire between 2004 and 2010. The PSQ Questionnaire was completed at 6 months post operation by all patients returning for a final check appointment. For the purposes of this study, there were no selection criteria beyond having undergone elective podiatric surgery and completed the PSQ.

The questionnaire was typically completed in the outpatient waiting room either immediately before or immediately following a clinic consultation. The questionnaires were entered onto an Access database by a team of healthcare assistants. The free text answers to question one were stored within an Access table.

The data was exported to an Excel spreadsheet for further analysis. A qualitative approach was adopted in order to categorise answers to Question one. An initial review of the free text answers identified four key responses which occurred repeatedly. These responses were labelled: Pain; Shoe Fitting; Activity; Cosmesis. Within each of these responses a number of key words were identified, these are listed in Table 1 . The individual answers to question one were then reviewed again. A number of void answers were identified and discarded. The remainder were then placed in each of the four responses and an additional fifth theme; 'Other', which was used for miscellaneous answers. A large number of patients stated multiple expectations and so key words from any of the four response categories were determined and counted accordingly for each patient. The data relating to responses was categorical and so descriptive statistics were applied throughout.

\section{Results}

Between January 2004 and September 2010, a total of 2910 unique surgical admissions were entered onto the PASCOM audit database. The majority of patients were female $(83.3 \%)$ and the age range was $14-95$ years. The admissions gave rise to 5312 surgical procedures. All admissions were for day care surgery and the majority (99\%) were performed under local anaesthesia. Seven podiatric surgeons and one podiatrist (non incision nail surgery procedures) contributed to the database.

Not all patients who underwent surgery subsequently completed the PSQ questionnaire, as a result a total of 1869 questionnaires were returned with the remaining $35.8 \%$ unaccounted for. As stated above, question one requires a text input. A total of 1458 returned questionnaires had some kind of input or wording entered for question one, while 411 were blank. A further 28 questionnaires were null and void where patient's had used the text box to make an unrelated comment or ask a question. This left a total of 1430 questionnaires available for further analysis.

Table 2 lists the number of counts recorded for expectations within each of the 5 response categories. A total of 2278 counts were recorded, 1191 counts (52.3\%) were recorded for the Pain response while footwear and mobility accounted for $16.6 \%$ and $16.4 \%$ respectively. Cosmesis counts occurred less commonly accounting for $12.2 \%$. A small number of counts (2.5\%) did not fit comfortably into any of the 4 main categories.

709 patients $(49.6 \%)$ entered only a single expectation in the question one text box. 599 patients (41.9\%) entered two expectations, 114 patients (8\%) entered three expectations and 7 patients $(0.5 \%)$ entered 4 expectations. Table 3 presents a summary of the responses for patients who entered only a single expectation. Again, pain relief was the dominant expectation accounting for $515(72.6 \%)$ patients who provided only 
Table 1 Patient expectation responses and example descriptors

\begin{tabular}{llll}
\hline Pain & Mobility & Shoe fitting & Cosmesis \\
\hline Pain free/relief/reduced & Past times & Wear normal shoes & Deformity \\
Relief & Walking -easier & Stylish footwear/shoes & Ugly \\
Discomfort & Walking - better & Better fitting shoes & A straight(er) toe(s) \\
Comfort & Walking correctly & Shoe fit & Alignment \\
Rubbing & Dancing & Comfortable shoes & Lump/Bump \\
Irritation & Running & Wear a full shoe & Unsightly \\
Throbbing & Movement & Wear shoes properly & Nicer looking \\
Ache & Mobility & & Removal of... \\
& & & Curled \\
& & & Crossing over \\
\hline
\end{tabular}

one response. Although cosmesis was the least common response overall, it was the second most common singular expectation accounting for 77 (5.4\%) responses.

Owing to the predominance of pain responses, we chose to further evaluate all questionnaires where an expectation of pain relief was recorded (Table 4). Pain responses were found in 1190 (83\%) of the 1430 returned questionnaires. $36.1 \%$ of the cohort expected nothing more than pain relief, $16.6 \%$ expected pain relief and improved mobility, while $14.2 \%$ expected pain relief, and improved shoe fitting. The remaining $17.2 \%$ of patients who expected pain relief also had a number of additional expectations as detailed in Table 4.

\section{Discussion}

The current paper has summarised for the first time, the expectations of patients attending for podiatric surgery. By far the most important expectation was for pain relief with $83 \%$ of patients referring to pain and $36.1 \%$ of patients expecting nothing more than pain relief. Expectations relating to mobility and shoe fitting were also important considerations while cosmesis was a relatively uncommon expectation. These findings are not dissimilar to those of Schneider and Knhar who found that the main expectations following hallux valgus surgery were to be pain free and able to wear a normal shoe [22]. The small number of counts for cosmesis is perhaps surprising given that most podiatric surgery procedures attempt to correct deformity. Radl et al, on the contrary, argue that cosmesis is actually a significant

Table 2 Total counts for each response (percentage of all themes).

\begin{tabular}{llllll}
\hline Count & Pain & Mobility & $\begin{array}{l}\text { Shoe } \\
\text { fitting }\end{array}$ & Cosmesis & Other \\
\hline Yes & 1190 & 374 & $379(16.6 \%)$ & 277 & 57 \\
& $(52.2 \%)$ & $(16.4 \%)$ & & $(12.2 \%)$ & $(2.6 \%)$ \\
\hline No & 239 & 1056 & 1153 & 1051 & 1373 \\
\hline
\end{tabular}

Total response counts $=2640$. Total cohort $=n .1430$. concern in hallux valgus surgery as opposed to many other orthopaedic procedures which address joint pain without consideration of cosmesis [26]. The current study did not assess expectation in relation to specific diagnoses but hallux valgus surgery accounted for 2162 (40\%) procedures.

It is interesting to note that for the most part, patient expectations fell into four clearly defined responses (pain relief, shoe fitting, mobility and cosmesis) and that these responses are not dissimilar to the domains measured by regional measures of health related quality of life. For example two foot health instruments; the Foot Health Status Questionnaire (FHSQ)[27]; and the Manchester Oxford Foot questionnaire (MOXFQ)[28], both include domains relevant to pain, mobility and foot wear while the MOXFQ additionally makes specific reference to cosmesis. The current paper provides further support for the validity of the quality of life domains measured by both the FHSQ and MOXFQ.

Increasingly clinicians are relying on patient reported outcome measures which provide a validated and objective method of assessing a patient's health related quality of life prior to and following intervention. These instruments provide an invaluable insight into the impact of a given foot pathology on a patient's quality of life and in doing so may indirectly measure a patient's expectations and satisfaction. Bennet stated that health related quality

Table 3 Summary of counts for patients reporting a single expectation and percentage of total cohort ( $n=1430$ )

\begin{tabular}{lll}
\hline Singular complaints & Count & Percentage \\
\hline Pain & 515 & $36.1 \%$ \\
Cosmesis & 77 & $5.4 \%$ \\
Other & 45 & $3.1 \%$ \\
Mobility & 37 & $2.6 \%$ \\
Shoes & 35 & $2.4 \%$ \\
\hline Total & $\mathbf{7 0 9}$ & \\
\hline
\end{tabular}


Table 4 Summary of all pain response combinations and percentage of total cohort $(n=1430)$

\begin{tabular}{lrr}
\hline Pain response combinations & Count & Percentage \\
\hline Total Pain Counts (any combination) & $\mathbf{1 1 9 0}$ & $\mathbf{8 3 \%}$ \\
\hline pain & 515 & $36.1 \%$ \\
Pain; mobility & 238 & $16.6 \%$ \\
Pain; shoes & 203 & $14.2 \%$ \\
Pain; cosmesis & 107 & $7.5 \%$ \\
Pain; mobility; shoes & 58 & $4.1 \%$ \\
Pain; cosmesis; shoes & 36 & $2.5 \%$ \\
Pain; mobility; cosmesis & 18 & $1.3 \%$ \\
Pain; mobility; cosmesis; shoes & 7 & $0.5 \%$ \\
Pain; other & 6 & $0.42 \%$ \\
Pain, cosmesis, other & 2 & $0.1 \%$ \\
\hline
\end{tabular}

of life (HRQOL) should be measured in preference to patient satisfaction [27]. On the contrary, Carr et al argue that HRQOL is actually defined as the gap between expectation of health and the patient's experience of health $[4,29]$. Patient expectations are then related to and may influence HRQOL, yet none of the current HRQOL instruments ask the question; 'what do you hope to gain from your treatment?' If patient expectations of healthcare are not considered, the planning of treatment appropriate to the patient's needs could be compromised.

Carr et al go further stating that HRQOL instruments cannot distinguish between changes in experience of disease and changes in expectation of health [4]. Patients will compare their personal experience and the experience of others to their own expectations of health when completing a HRQOL instrument [4,5]; The point where a given HRQOL scores start (prior to treatment) and finish (following intervention) is influenced by patient expectation and so if such instruments are used to study outcomes, arguably patient expectations must also be recorded [4].

Measurement of patient expectations and satisfaction is, one could argue a more personal or qualitative approach to assessing outcomes. Recording patient expectations of treatment allows clinicians' to better understand the impact of a disease process from the patient's perspective. This understanding may then be used to guide treatment planning. There is evidence that patient expectations are important in determining the success or failure of intervention and so expectations should arguably be determined prior to intervention. Patient expectations as presented during the initial consultation may actually influence treatment decisions although doctors perceptions have been found to be more important than patient expectation in determining treatment [30]. Therefore disparity between clinical perception of a patient's expectation of treatment and the patient's actual expectation may be a cause of poor outcomes [30].

It cannot be denied that question one of the PSQ offers patients an opportunity to describe their expectations. It is though important to note that this determination of patient expectation only occurs retrospectively. The patient is asked to consider their expectations sometime after treatment. This creates a significant methodological problem. However Kadezielski et al found that pre operative expectations did not correlate with post operative satisfaction in the context of carpal tunnel surgery [7]. Nonetheless, the current study cannot determine what effect treatment, recovery and overall perioperative experience have on how the patient answers question one.

We do know from previous work that patient expectations are at least partly a consequence of prior experience, the experience of others, environmental factors, personality type and the treatment in itself $[4,26]$. Similarly others have found an association between poor outcomes and unrealistic expectations $[5,7,13,14]$. In the current study, we did not attempt to quantify whether patient expectations were unrealistic. Rather we have presented expectations as found. This paper also chose to review patient expectations across all podiatric surgery procedures as a single cohort whereas previous authors have concentrated on hallux valgus surgery alone $[21,22,26]$. There may be some value in analysing expectations relating to specific diagnoses, Delgado et al found that expectations vary depending on the presenting disease process in the setting of primary care [31].

Data collection for this study started before the introduction of patient reported outcomes into routine podiatric surgery practice in the UK. Analysing patient expectation in relation to pre operative HRQOL scores and measuring patient satisfaction in relation to post operative HRQOL scores would be a valuable endeavour. We cannot, at this time, be certain of what impact (if any) patient expectation has on health related quality of life in the context of foot surgery. Although we now have a basic understanding of expectation we do not know what factors, such as quality of life or surgical complications, may influence the clinician's ability to meet a patient's expectations and further work is required to determine why expectations have or have not been met and to determine the impact multiple expectations may have on surgical outcomes.

There were a number of weaknesses with the study design. Perhaps most significant of these is retrospective data collection. Patients were asked to describe their pre operative expectations of surgery, 6 months following treatment. There was no attempt to correlate this finding with a pre operative measure of expectation. Retrospective data collection also resulted in a significant loss 
to follow up. During the study period 2910 patients attended for surgery but only 1869 questionnaires were returned. We do not know what effect this loss to follow up had on the actual expectation responses.

Compounding the loss to follow up, the design of the questionnaire itself resulted in a further data loss with only 1430 patients completing question 1 . This data loss could be minimised by formatting question 1 as per the remaining PSQ questions; utilising a list of responses and tick boxes as opposed to a free text.

\section{Conclusions}

This paper demonstrates the expectations of a large cohort of podiatric surgery patients. For the most part patients expect pain relief, improved mobility and improved shoe fitting, while a small number of patients also expect a cosmetic improvement. Further research is required to determine the relationship between patient expectation and health related quality of life, and to determine whether podiatric surgery is successful in addressing the expectations of patients.

\section{Additional material}

\section{Additional file 1: Patient Satisfaction Questionnaire}

\section{Acknowledgements \\ Thank you to Jackie Ludlam and Mavis Clark who both dedicated many hours to maintaining the database.}

\section{Author details}

'Department of Podiatric Surgery, Doncaster and Bassetlaw Hospitals NHS Foundation Trust, Cantley Health Centre, Middleham Road, Cantley Doncaster, South Yorkshire, DN4 6ED, UK. ²Department of Podiatric Surgery, Park House Health and Social Care Centre, 61 Burton Road, Carlton, Nottingham, NG4 3DQ, UK.

\section{Authors' contributions}

ANW took responsibility for the implementation of PASCOM in Doncaster, England and over saw data collection. The paper was jointly devised by AJM and ANW, AJM prepared the manuscript and both authors reviewed and edited the final manuscript prior to submission.

\section{Competing interests}

AJM is a current member of the PASCOM working party of the Society of Chiropodists and Podiatrists, UK and is directly involved with developmental work relating to outcome measurement. Neither author received a financial reward for the production of this manuscript.

Received: 25 May 2011 Accepted: 6 December 2011

Published: 6 December 2011

\section{References}

1. Maher AJ, Kilmartin T: Patient-reported outcomes: a new direction for podiatric surgery. Podiatry Now 2010, 13:36-37.

2. Fitzpatrick R: Surveys of patient satisfaction: I-Designing a questionnaire and conducting a survey. BMJ 1991, 302:1129-1132.

3. Shelton PJ: Measuring and Improving Patient Satisfaction Maryland: Aspen Publishers; 2000
4. Carr AJ, Gibson B, Robinson PG: Measuring quality of life: Is quality of life determined by expectations or experience? BMJ 2001, 322:1240-1243.

5. McKinley RK, Stevenson K, Adams S, Manku-Scott TK: Meeting patient expectations of care: the major determinant of satisfaction with out-ofhours primary medical care? Fam Pract 2002, 19:333-338.

6. Mahomed NN, Liang MH, Cook EF, Daltroy LH, Fortin PR, Fossel AH, Katz JN: The importance of patient expectations in predicting functional outcomes after total joint arthroplasty. J Rheumatol 2002, 29:1273-1279.

7. Kadzielski J, Malhotra LR, Zurakowski D, Lee SG, Jupiter JB, Ring D: Evaluation of preoperative expectations and patient satisfaction after carpal tunnel release. J Hand Surg Am 2008, 33:1783-1788.

8. Mancuso CA, Salvati EA, Johanson NA, Peterson MG, Charlson ME: Patients' expectations and satisfaction with total hip arthroplasty. J Arthroplasty 1997, 12:387-396.

9. Haworth RJ, Hopkins J, Ells P, Ackroyd CE, Mowat AG: Expectations and outcome of total hip replacement. Rheumatol Rehabil 1981, 20:65-70.

10. Engel C, Hamilton NA, Potter PT, Zautra AJ: Impact of two types of expectancy on recovery from total knee replacement surgery (TKR) in adults with osteoarthritis. Behav Med 2004, 30:113-123.

11. Henn RF, Kang L, Tashjian RZ, Green A: Patients' preoperative expectations predict the outcome of rotator cuff repair. J Bone Joint Surg Am 2007, 89:1913-1919.

12. Iversen $M D$, Daltroy $L H$, Fossel $A H$, Katz JN: The prognostic importance of patient pre-operative expectations of surgery for lumbar spinal stenosis. Patient Educ Couns 1998, 34:169-178.

13. Wheelock I, Peterson C, Buchtel HA: Presurgery expectations, postsurgery satisfaction, and psychosocial adjustment after epilepsy surgery. Epilepsia 1998, 39:487-494.

14. Orbell S, Johnston M, Rowley D, Espley A, Davey P: Cognitive representations of illness and functional and affective adjustment following surgery for osteoarthritis. Soc Sci Med 1998, 47:93-102.

15. Kilmartin TE, O'Kane C: Combined rotation scarf and Akin osteotomies for hallux valgus: a patient focussed 9 year follow up of 50 patients. J Foot Ankle Res 2010, 3:2.

16. Maher AJ, Kilmartin TE: Scarf osteotomy for correction of Tailor's bunion: mid- to long-term followup. Foot Ankle Int 2010, 31:676-682.

17. Maher AJ, Metcalfe SA: A report of UK experience in 917 cases of day care foot surgery using a validated outcome tool. Foot 2009, 19:101-106.

18. Ashford RL, Vogiatzogloub F, Tollafield DR, Cassellad JP: A retrospective analysis of Swanson Silatic double-stemmed great toe implants eith titanium grommets following podiatric surgery for arthritic joint disease. Foot 2000, 10:69-74.

19. Tollafield DR: An audit of lesser metatarsal osteotomy by capital proximal displacement (Weil osteotomy). British Journal of Podiatry 2001, 4:15-19.

20. Maher AJ, Metcalfe SA: First MTP joint arthrodesis for the treatment of hallux rigidus: results of 29 consecutive cases using the foot health status questionnaire validated measurement tool. Foot 2008, 18:123-130.

21. Tai CC, Ridgeway S, Ramachandran M, Ng VA, Devic N, Singh D: Patient expectations for hallux valgus surgery. J Orthop Surg (Hong Kong) 2008, 16:91-95.

22. Schneider W, Knahr K: Surgery for hallux valgus. The expectations of patients and surgeons. Int Orthop 2001, 25:382-385.

23. Tollafield DR, Parmar DG: Setting standards for day care foot surgery. A quniquenial review. Br J Podiatr Med Surg 1994, 6:7-20.

24. Rudge G, Tollafield DR: A critical assessment of a new evaluation tool for podiatric surgical outcome analysis. Br J Podiatr 2003, 6:100-110.

25. Taylor NG, Tollafield DR, Rees S: Does patient satisfaction with foot surgery change over time? Foot 2008, 18:68-74

26. Radl R, Leithner A, Zacherl M, Lackner U, Egger J, Windhager R: The influence of personality traits on the subjective outcome of operative hallux valgus correction. Int Orthop 2004, 28:303-306.

27. Bennett PJ, Patterson C, Wearing S, Baglioni T: Development and validation of a questionnaire designed to measure foot-health status. Am Podiatr Med Assoc 1998, 88:419-428.

28. Dawson J, Coffey J, Doll H, Lavis G, Cooke P, Herron M, Jenkinson C: A patient-based questionnaire to assess outcomes of foot surgery: validation in the context of surgery for hallux valgus. Qual Life Res 2006, 15:1211-1222

29. Calman KC: Quality of life in cancer patients-an hypothesis. J Med Ethics 1984, 10:124-127.

30. Britten N: Patients' expectations of consultations. BMJ 2004, 328:416-417. 
31. Delgado A, Andres Lopez-Fernandez L, de Dios Luna J, Gil N, Jimenez M, Puga A: Patient expectations are not always the same. J Epidemiol Community Health 2008, 62:427-434.

doi:10.1186/1757-1146-4-27

Cite this article as: Wilkinson and Maher: Patient expectations of podiatric surgery in the United Kingdom. Journal of Foot and Ankle Research 2011 4:27.

Submit your next manuscript to BioMed Central and take full advantage of:

- Convenient online submission

- Thorough peer review

- No space constraints or color figure charges

- Immediate publication on acceptance

- Inclusion in PubMed, CAS, Scopus and Google Scholar

- Research which is freely available for redistribution

Submit your manuscript at www.biomedcentral.com/submit 